\title{
Evaluation of Dislocation Density in a Mg-Al-Mn-Ca Alloy Determined by X-ray Diffractometry and Transmission Electron Microscopy
}

\author{
Takashi Shintani*1, Yoshinori Murata*2, Yoshihiro Terada and Masahiko Morinaga \\ Department of Materials, Physics and Energy Engineering, Graduate School of Engineering, \\ Nagoya University, Nagoya 464-8603, Japan
}

\begin{abstract}
Metallic materials suffering deformation store elastic strain. Evaluation of this strain energy is important for understanding the mechanical and physical properties of the materials. Although direct evaluation of the stored energy is difficult, it can be evaluated by determining the defect energy of dislocations induced by the deformation. Thus, a practicable method of evaluating the strain energy is to measure the dislocation density in metallic materials. The average and representative dislocation density can be estimated by X-ray diffraction (XRD) analysis. We have estimated the dislocation density of a magnesium alloy with hexagonal crystals by a modified Warren-Averbach analysis based on a modified Williamson-Hall plot using XRD profiles. The dislocation density value obtained by this method agrees with those reported previously. We found that the modified Warren-Averbach method is still a powerful method for evaluating the dislocation density in hexagonal crystals. [doi:10.2320/matertrans.M2010021]
\end{abstract}

(Received January 21, 2010; Accepted March 8, 2010; Published April 21, 2010)

Keywords: $X$-ray diffraction, transmission electron microscopy, magnesium alloys, dislocations

\section{Introduction}

In the past decade, owing to its extremely light weight, magnesium alloys have been used in the automotive industry for improving fuel efficiency through vehicle mass reduction. However, low temperature capability of the alloys has restricted their practical use. It is therefore desirable to develop magnesium alloys with high temperature capability. ${ }^{1)}$

$\mathrm{AM}$ (Mg-Al-Mn) series alloys are commonly used in automobiles because of a good combination of their mechanical properties, corrosion resistance and die-castability. Calcium is a cost-effective, lighter alternative to rare-earth elements for improving the resistance to high temperatures of AM series alloys. ${ }^{1)}$ The creep strength of AM50 die-cast alloys has been improved by the additions of calcium. $\mathrm{AM} 50+x \mathrm{Ca}(x=0.47,0.95$ and 1.72 mass $\%)$ die-cast alloys are superior in high temperature capability to AM50, as reported by Itoh et al. ${ }^{1)}$ Also, the value of the dislocation density of AX52 (AM50 + 1.72Ca) die-cast alloy has been measured with transmission electron microscopy (TEM) techniques. The chemical composition of AX52 is shown in Table 1. ${ }^{1)}$

The dislocation density can be measured by direct methods, such as TEM techniques, and by indirect methods, such as X-ray diffraction (XRD) or neutron techniques. These experimental techniques are complementary. Direct techniques can reveal microstructural information over extremely small areas of samples, whereas the latter two techniques can reveal average and representative data. However, the sample preparation procedures for TEM studies are more difficult and complicated than those for XRD or neutron studies. Furthermore, it is known that the small thickness of TEM samples results in low dislocation density.

\footnotetext{
${ }^{* 1}$ Graduate Student, Nagoya University

${ }^{* 2}$ Corresponding author, E-mail: murata@numse.nagoya-u.ac.jp
}

Table 1 Chemical composition of AX 52 die-cast alloy (mass\%)

\begin{tabular}{cccc}
\hline $\mathrm{Al}$ & $\mathrm{Ca}$ & $\mathrm{Mn}$ & $\mathrm{Mg}$ \\
\hline 4.98 & 1.72 & 0.29 & bal. \\
\hline
\end{tabular}

In recent years, XRD profile analysis has been developed to reveal microstructural details, such as dislocation density and crystallite size. The measurement of microstructural details in hexagonal crystals is more difficult than in cubic crystals because of the complexity of their crystal structures. The multiple whole-profile (MWP) fitting procedure ${ }^{2)}$ is the principal analysis method for measuring microstructural details in hexagonal crystals, such as $\mathrm{Mg},{ }^{3,4)} \mathrm{Ti}^{5,6)}$ and $\mathrm{Zr}^{7 \text { ) }}$ However, the principle of the procedure is complicated, and also the results depend strongly on the input data with arbitrary properties. The purpose of this study is to apply a simpler method proposed originally by Ungár et al. ${ }^{8)}$ to analyse microstructural details in Mg-based alloys. The results are compared with those obtained by TEM and their validity is discussed.

\section{Method of Analysing X-ray Diffraction Profiles}

\subsection{Calculation of $\overline{b^{2} C}$}

In hexagonal crystals, the average contrast factor of dislocation, $\overline{C_{h k . l}}$ corresponding to (hk.l) reflections can be written as ${ }^{9)}$

$$
\overline{C_{h k . l}}=\overline{C_{h k .0}}\left(1+q_{1} x+q_{2} x^{2}\right),
$$

where $x=(2 / 3)(l / g a)^{2}$, and $q_{1}$ and $q_{2}$ are parameters that depend on the elastic properties of the material. $\overline{C_{h k .0}}$ is the average contrast factor corresponding to (hk.0) reflections. $a$ and $l$ are the lattice constant in the basal plane and the last index of the (hk.l) reflection, respectively. $g$ is the diffraction vector, which is a function of the fourth-order invariant of the (hk.l) indices and the lattice constants. Hereafter, $\overline{C_{h k . l}}$ is represented as $\bar{C}$ for simplicity. 
Table 2 The most common slip systems in hexagonal crystals.

(a) Edge dislocations

\begin{tabular}{ccccc}
\hline $\begin{array}{c}\text { Major slip } \\
\text { systems }\end{array}$ & Sub-slip systems & Burgers vector & Slip plane & $\begin{array}{c}\text { Burgers vector } \\
\text { types }\end{array}$ \\
\hline Basal & BE & $\langle 2 \overline{1} \overline{1} 0\rangle$ & $\{0001\}$ & $\boldsymbol{a}$ \\
\hline Prismatic & PrE & $\langle\overline{2} 110\rangle$ & $\{01 \overline{1} 0\}$ & $\boldsymbol{a}$ \\
\hline & PrE2 & $\langle 0001\rangle$ & $\{01 \overline{1} 0\}$ & $\boldsymbol{c}$ \\
\hline Pyramidal & PrE3 & $\langle\overline{2} 113\rangle$ & $\{01 \overline{1} 0\}$ & $\boldsymbol{c}+\boldsymbol{a}$ \\
\hline & PyE & $\langle\overline{1} 2 \overline{1} 0\rangle$ & $\{10 \overline{1} 1\}$ & $\boldsymbol{a}$ \\
\hline & PyE3 & $\langle\overline{2} 113\rangle$ & $\{2 \overline{1} \overline{1} 2\}$ & $\boldsymbol{c}+\boldsymbol{a}$ \\
\hline & PyE4 & $\langle\overline{2} 113\rangle$ & $\{11 \overline{2} 1\}$ & $\boldsymbol{c}+\boldsymbol{a}$ \\
\hline
\end{tabular}

(b) Screw dislocations

\begin{tabular}{ccc}
\hline Slip systems & Burgers vector & $\begin{array}{c}\text { Burgers vector } \\
\text { types }\end{array}$ \\
\hline S1 & $\langle 2 \overline{1} \overline{1} 0\rangle$ & $\boldsymbol{a}$ \\
\hline S2 & $\langle\overline{2} 113\rangle$ & $\boldsymbol{c}+\boldsymbol{a}$ \\
\hline S3 & $\langle 0001\rangle$ & $\boldsymbol{c}$ \\
\hline
\end{tabular}

$\bar{b}^{2}{ }^{(m)}$ is the measured $\overline{b^{2} C}$ of the sample. $b$ represents the magnitude of Burgers vector. Table 2 shows the most common slip systems in hexagonal crystals. There are three types of fundamental Burgers vectors represented as a type, $\mathbf{c}$ type and $\mathbf{c}+\mathbf{a}$ type in hexagonal crystals. In the analysis, therefore, averaged value of the magnitude of Burgers vector is used. $\overline{b^{2}}$ represents the averaged value of square $b$.

${\overline{b^{2} C}}^{(m)}$ can be written as ${ }^{9)}$

$$
{\overline{b^{2} C}}^{(m)}=\sum_{i=1}^{N} f_{i} \bar{C}^{(i)} b_{i}^{2},
$$

where $N$ is the number of different sub-slip systems. In the case of hexagonal crystals, $N$ is equal to 11 , as shown in Table 2.5) $\bar{C}^{(i)}$ and $b_{i}$ are the average contrast factor and the Burgers vector corresponding to the $i$ th sub-slip system, respectively. $f_{i}$ are the fractions of the particular sub-slip systems, where $\sum_{i=1}^{N} f_{i}=1$ and $f_{i} \geq 0$. For a hexagonal crystal structure, eq. (2) can be written using three types of fundamental Burgers vectors, defined as $b_{1}=1 / 3\langle-2110\rangle$ (a type), $b_{2}=1 / 3\langle 0001\rangle$ (c type) and $b_{3}=1 / 3\langle-2113\rangle$ $(\mathbf{c}+\mathbf{a}$ type $)$,

$$
{\overline{b^{2} C_{h k . l}}}^{(m)}=b_{1}^{2} \sum_{i=1}^{N\langle a\rangle} f_{i} \overline{C^{(i)}}+b_{2}^{2} \sum_{j=1}^{N\langle c\rangle} f_{j} \overline{C^{(j)}}+b_{3}^{2} \sum_{n=1}^{N\langle c+a\rangle} f_{n} \overline{C^{(n)}}
$$

where $N\langle a\rangle, N\langle c\rangle$ and $N\langle c+a\rangle$ are the number of sub-slip systems with Burgers vector types a, $\mathbf{c}$ and $\mathbf{c}+\mathbf{a}$, respectively. The values of $f_{i}$ were determined by the relative fractions of the population of the Burgers vectors types, a, c and $\mathbf{c}+\mathbf{a}$, on the basis of the number of sub-slip systems. As a result, eq. (3) can be written as ${ }^{5,9)}$

$$
{\overline{b^{2} C_{h k . l}}}^{(m)}=\sum_{i=1}^{3} h_{i} \bar{C}^{(i)} b_{i}^{2},
$$

where $h_{i}$ are the fractions of the dislocation population in the sample with the same Burgers vector, $b_{a}, b_{c}$ and $b_{c+a}$. Now, $\bar{C}^{(i)}$ are the average contrast factors over the sub-slip systems for the same Burgers vector types. Substituting eq. (1) in eq. (4), the following three equations are obtained:

$$
\begin{aligned}
& q_{1}^{(m)}=\frac{1}{P} \sum_{i=1}^{3} h_{i} \overline{C_{h k .0}}{ }^{(i)} b_{i}^{2} q_{1}^{(i)} \\
& q_{2}^{(m)}=\frac{1}{P} \sum_{i=1}^{3} h_{i} \overline{C_{h k .0}}{ }^{(i)} b_{i}^{2} q_{2}^{(i)} \\
& \sum_{i=1}^{3} h_{i}=1
\end{aligned}
$$

where

$$
\begin{aligned}
& P=\sum_{i=1}^{3} h_{i}{\overline{C_{h k .0}}}^{(i)} b_{i}^{2}={\overline{b^{2} C_{h k .0}}}^{(m)}, \\
& 0 \leq h_{i} \leq 1 .
\end{aligned}
$$

$q_{1}{ }^{(m)}$ and $q_{2}{ }^{(m)}$ are the measured $q_{1}$ and $q_{2}$, respectively, which are determined from the modified Williamson-Hall plot mentioned in section 2.2. They can be obtained from the XRD profiles. $\overline{C_{h k .0}}{ }^{(i)}, q_{1}{ }^{(i)}$ and $q_{2}{ }^{(i)}$ are the numerically calculated values for all sub-slip systems.

To evaluate the three unknowns, $h_{a}, h_{c}$ and $h_{c+a}$ the values of the abovementioned parameters $\frac{C_{h k .0}{ }^{(i)}}{{ }^{2}}, q_{1}{ }^{(i)}$ and $q_{2}{ }^{(i)}$ are required for solving the simultaneous equations of eq. (5). They were published previously for the most common subslip systems. ${ }^{9)}$ Using the average values of $h_{a}, h_{c}$ and $h_{c+a}$, $\overline{b^{2} C}$ can be determined from eq. (6).

\subsection{Calculation of the values of $q_{1}{ }^{(m)}$ and $q_{2}{ }^{(m)}$ by the modified Williamson-Hall plot}

The value of the full width at half-maximum (FWHM) obtained from each peak is substituted into the following modified Williamson-Hall equation: ${ }^{10)}$

$$
\Delta K \cong \frac{0.9}{D}+\left(\frac{\pi M^{2} b^{2}}{2}\right)^{1 / 2} \rho^{1 / 2} K \bar{C}^{1 / 2}+O\left(K^{2} \bar{C}\right),
$$

where $K=2 \sin \theta / \lambda$ and $\Delta K=2 \cos \theta(\Delta \theta) / \lambda$. Here, $\Delta \theta, \theta$ and $\lambda$ are the FWHM, diffraction angle and wavelength of the $\mathrm{X}$-rays, respectively. In the case of $\mathrm{Cu}, \lambda=0.15405 \mathrm{~nm}$. $D, \rho$ and $b$ are the average particle size, the dislocation density and the magnitude of the Burgers vector, respectively. $M$ is a constant depending on both the effective outer cut-off radius of dislocations and the dislocation density. $O$ stands for higher-order terms in $K \bar{C}^{1 / 2}$. Equation (8) is converted into the quadratic form, in which its high-order term becomes negligible. Modifying eq. (1) into the quadratic form, the following equation is obtained:

$$
\left[(\Delta K)^{2}-\alpha\right] / K^{2} \cong \beta \overline{C_{h k .0}}\left(1+q_{1} x+q_{2} x^{2}\right),
$$

where $\alpha=(0.9 / D)^{2}$ and $\beta=\pi M^{2} b^{2} \rho / 2$. The value of $\alpha$ is determined while keeping the left-hand side of eq. (9) as a quadratic function of $x$, as shown in Fig. 1(a). As a result, the measured values of $q_{1}{ }^{(m)}$ and $q_{2}{ }^{(m)}$ are obtained from the coefficients of $x$ in the quadratic function.

\subsection{Evaluation of the dislocation density by the modified Warren-Averbach analysis}

The modified Warren-Averbach equation is written as ${ }^{11)}$ 

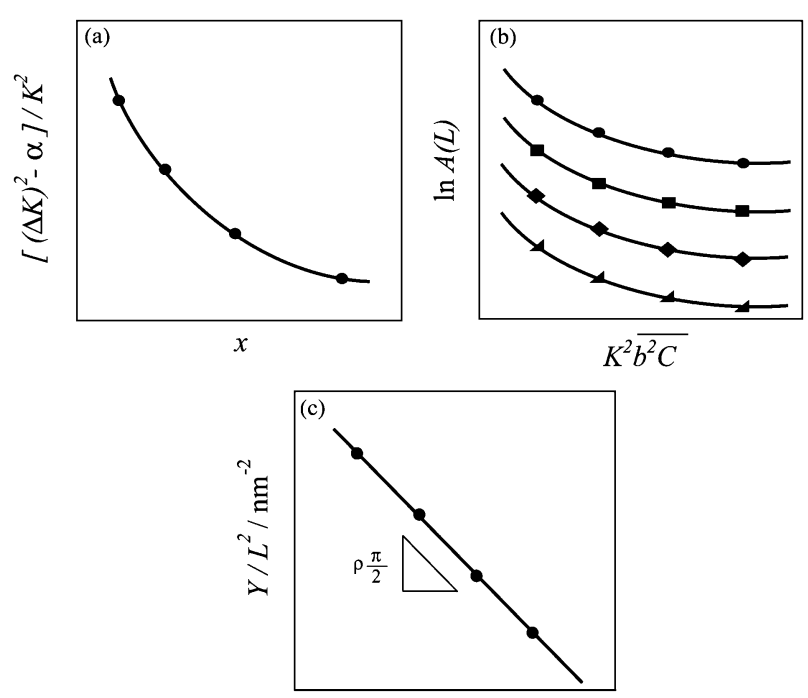

$\ln L$

Fig. 1 Schematic diagrams showing the method of analysis: (a) $\left[(\Delta K)^{2}-\right.$ $\alpha] / K^{2}$ versus $x$ plot following eq. (9); (b) the modified Warren-Averbach plot following eq. (11); (c) $Y / L^{2}$ versus $\ln L$.

$$
\begin{aligned}
\ln A(L) \cong & \ln A^{S}(L)-\rho \frac{\pi b^{2}}{2} L^{2} \ln \left(\frac{R_{e}}{L}\right)\left(K^{2} \bar{C}\right) \\
& +Q\left(\frac{\pi b^{2}}{2}\right)^{2} L^{4} \ln \left(\frac{R_{1}}{L}\right) \ln \left(\frac{R_{2}}{L}\right)\left(K^{4} \bar{C}^{2}\right) .
\end{aligned}
$$

For hexagonal crystals, eq. (10) can be arranged as

$$
\begin{aligned}
\ln A(L) \cong & \ln A^{S}(L)-\rho \frac{\pi}{2} L^{2} \ln \left(\frac{R_{e}}{L}\right)\left(K^{2} \overline{b^{2} C}\right) \\
& +Q\left(\frac{\pi}{2}\right)^{2} L^{4} \ln \left(\frac{R_{1}}{L}\right) \ln \left(\frac{R_{2}}{L}\right)\left(K^{4} \bar{b}^{2} C^{2}\right) .
\end{aligned}
$$

Here, $A(L)$ is the real part of the Fourier coefficients of the XRD profiles. Superscript $S$ in the term $\ln A(L)$ indicates the crystallite size, and $L$ is the Fourier variable. $L$ is defined as $^{12)}$

$$
L=n a_{3},
$$

where $a_{3}=\lambda /\left\{2\left(\sin \theta_{2}-\sin \theta_{1}\right)\right\}, n$ are integers starting from 0 and $\left(\theta_{2}-\theta_{1}\right)$ is the angular range of the measured diffraction profiles. ${ }^{12)} R_{e}$ is the effective outer cut-off radius of dislocations. $Q, R_{1}$ and $R_{2}$ are all constants.

$\ln A(L)$ can be considered as a function of $K^{2} \overline{b^{2} C}$ from eq. (11), and hence the dislocation density is determined from the $\ln A(L)$ versus $K^{2}{\overline{b^{2} C_{h k .0}}}^{(m)}$ plot, as shown in Fig. 1(b), as the coefficient of the second term in eq. (11) $Y$, which is arranged as

$$
\frac{Y}{L^{2}}=\rho \frac{\pi}{2} \ln R_{e}-\rho \frac{\pi}{2} \ln L
$$

The value of $\rho$ is evaluated from the gradient of the linear relationship between $Y / L^{2}$ and $\ln L$, as shown in Fig. 1(c).

\section{Experimental Procedures}

A sample of AX52 die-cast alloy was cut into the proper size and polished mechanically with emery papers down to $\# 2000$, followed by buff polishing with $\mathrm{Al}_{2} \mathrm{O}_{3}$ powders

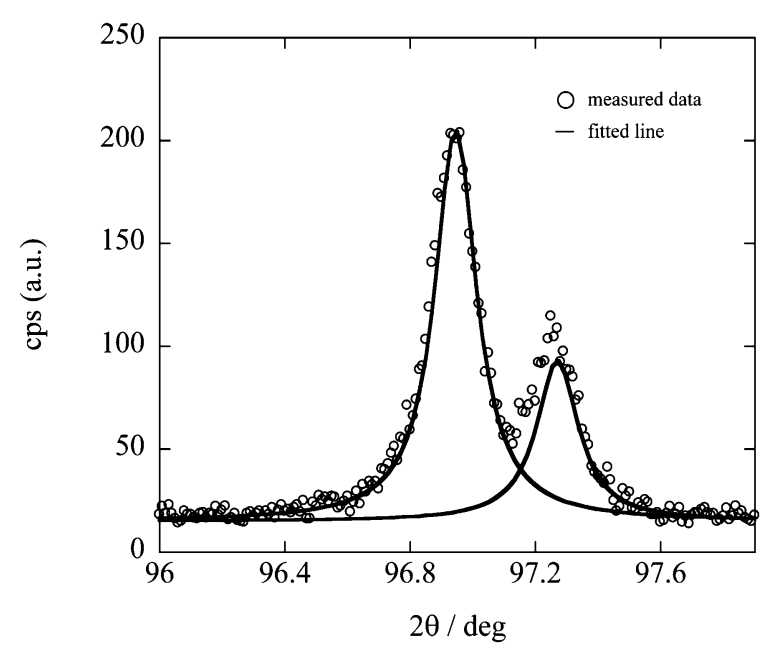

Fig. 2 Measured data and deconvolution lines using a Lorenz function of the (21.1) reflection.

Table 3 The diffraction angles and FWHM of the diffraction profiles used in this study.

\begin{tabular}{ccc}
\hline Bragg reflections & $2 \theta / \mathrm{deg}$ & FWHM/deg \\
\hline 00.2 & 34.47 & $3.97 \times 10^{-2}$ \\
\hline 10.1 & 36.76 & $4.32 \times 10^{-2}$ \\
\hline 11.0 & 57.71 & $6.22 \times 10^{-2}$ \\
\hline 20.0 & 67.50 & $7.17 \times 10^{-2}$ \\
\hline 20.1 & 70.20 & $7.46 \times 10^{-2}$ \\
\hline 21.1 & 97.15 & $1.15 \times 10^{-1}$ \\
\hline
\end{tabular}

down to $0.3 \mu \mathrm{m}$. Further, electropolishing was performed to remove the extra dislocations introduced into the sample surface by the earlier mechanical polishing.

For the XRD analysis, the diffraction profiles of the (00.2), (10.1), (11.0), (20.0), (20.1) and (21.1) reflections were measured with a conventional diffractometer (Rigaku UltimaIV X-ray diffractometer), using $\mathrm{Cu} \mathrm{K} \alpha_{1}$ and $\mathrm{K} \alpha_{2}$ radiation operating at $40 \mathrm{kV}$ and $40 \mathrm{~mA}$ at a scan speed of $0.25^{\circ} \mathrm{min}^{-1}$.

In this study, the modified Williamson-Hall as well as the modified Warren-Averbach plots were employed to obtain the microstructural details involved in dislocation density. ${ }^{9,10,13)}$ In these plots, the peaks obtained from only $\mathrm{Cu} \mathrm{K} \alpha_{1}$ radiation were needed. Therefore, each peak was separated into $\mathrm{K} \alpha_{1}$ radiation and $\mathrm{K} \alpha_{2}$ radiation using a Lorenz function, and the FWHM of all peaks were obtained. A representative result of peak deconvolution is shown in Fig. 2.

\section{Results and Discussion}

The XRD profile data obtained from AX52 alloy are listed in Table 3. Using these data, analysis was performed according to eqs. (8) and (9). As a result, $\alpha=4.29 \times$ $10^{-5} \mathrm{~nm}^{-2}, q_{1}{ }^{(m)}=-0.266$ and $q_{2}{ }^{(m)}=0.0184$ are obtained from the $\left[(\Delta K)^{2}-\alpha\right] / K^{2}$ versus $x$ plot, as shown in Fig. 3 . Although the plot data is localised, a smooth quadratic curve was obtained.

From the value of $\alpha$, the average particle size $D$ can be determined as: $D=137 \mathrm{~nm}$. The value of $D$ is apparent grain 


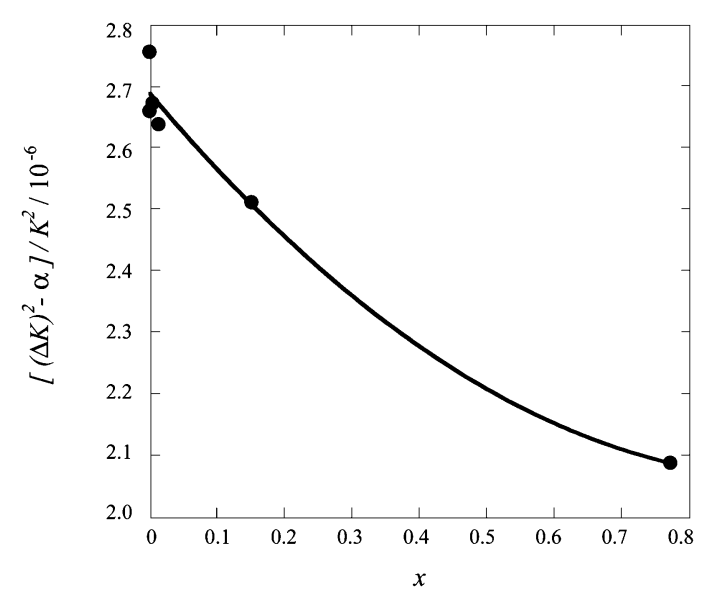

Fig. 3 The relationship between $\left[(\Delta K)^{2}-\alpha\right] / K^{2}$ and $x$ in eq. (9).

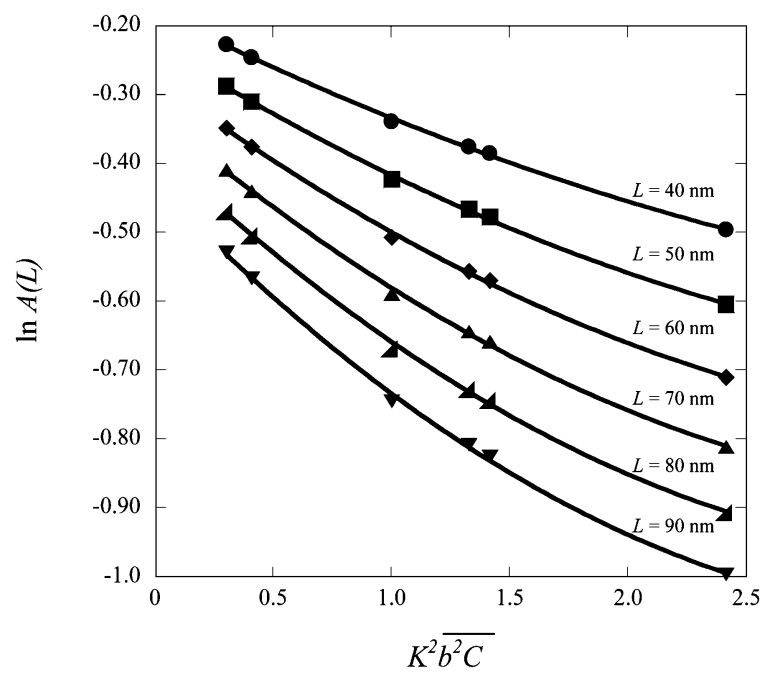

Fig. 4 The modified Warren-Averbach plot following eq. (11).

size measured from the modified Williamson-Hall plot. This value reflects the grain size but is not always identical to the real size. Therefore, it should be considered to be a measure of the grain size. ${ }^{14)}$

The values of $q_{1}{ }^{(m)}$ and $q_{2}{ }^{(m)}$ are substituted into eq. (5), and then the fractions $h_{i}$ of the three Burgers vector types are determined: $h_{a}=0.84, h_{c}=0.10$ and $h_{c+a}=0.06$. Using $h_{i}$, the modified Warren-Averbach plot was prepared according to eq. (11). The result is shown in Fig. 4. A series of quadratic curves was obtained using several $L$ values. As a result, $\rho=5.3 \times 10^{13} \mathrm{~m}^{-2}$ and $R_{e}=152 \mathrm{~nm}$ were estimated, as shown in Fig. 5.

To discuss the validity of the results obtained in this study and the method employed, values reported previously on the dislocation density of magnesium alloys ${ }^{1,4)}$ are listed in Table 4, together with the value obtained in this study.

Itoh et al. reported the dislocation density of AX52 diecast alloy, ${ }^{1)}$ which is the same alloy used in this study. They measured the density with TEM techniques, and their reported value of $\rho$ is smaller than that of this study. In general, it is known that some dislocations leave the specimen surface, and a dislocation-free zone is formed in a very thin layer at the surface. The thickness of conventional TEM samples is roughly several tens of $\mathrm{nm}$; hence, as

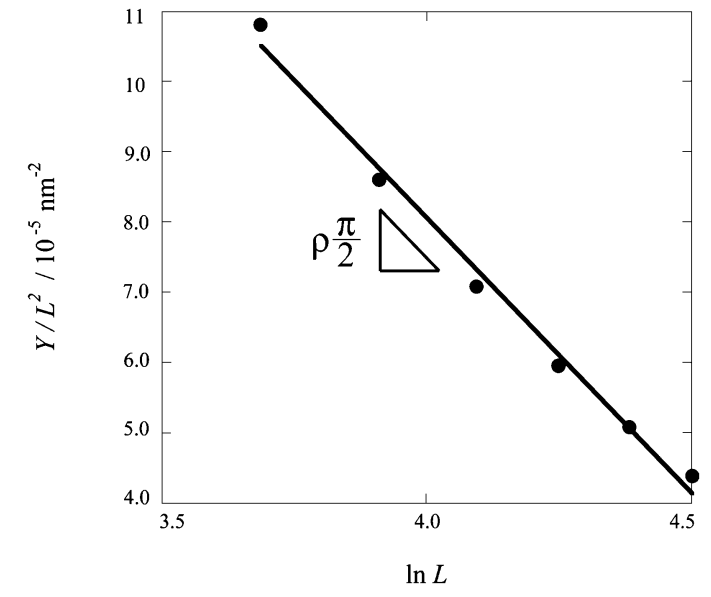

Fig. $5 Y / L^{2}$ versus $\ln L$ plot according to eq. (13). The gradient of the regression line provides the dislocation density. The effective outer cut-off radius of dislocations is also obtained from the intercept value of the horizontal scale.

Table 4 The comparison between the result of this study and others.

\begin{tabular}{cccc}
\hline & Specimen & Method of analysis & $\begin{array}{c}\text { Dislocation } \\
\text { density } \\
\rho / 10^{13} \mathrm{~m}^{-2}\end{array}$ \\
\hline $\begin{array}{c}\text { This } \\
\text { study }\end{array}$ & AX52 die-cast alloy & $\begin{array}{c}\text { modified Warren-Averbach } \\
\text { plot }\end{array}$ & 5.3 \\
\hline $\begin{array}{c}\text { Itoh's } \\
\text { study }\end{array}$ & AX52 die-cast alloy & TEM observation & 2.0 \\
\hline $\begin{array}{c}\text { Mathis's } \\
\text { study }\end{array}$ & $\begin{array}{c}\text { AZ91 alloy } \\
\text { (heat treated 18h } \\
\text { at 686 K) }\end{array}$ & MWP fitting procedure & 4.0 \\
\hline
\end{tabular}

mentioned in section 1 , the dislocation-free zone affects the number of dislocations and results in low density. Furthermore, in TEM contrasts, it is not necessarily the case that all dislocations can be measured, because of the extinction law in TEM observations. The true value of $\rho$ in the sample must be larger than that reported by Itoh et al. With this interpretation, our result is consistent with the earlier results obtained by TEM observation and is considered to be practicable.

Máthis et al. calculated the dislocation density of AZ91 alloys heat treated for $18 \mathrm{~h}$ at $686 \mathrm{~K}^{4)}$ by XRD analysis. Their reported value of $\rho$ is very close to that in this study. They used the MWP fitting procedure, which is the principal analysis method for obtaining the value of the dislocation density from XRD profiles. ${ }^{2)}$ Although the alloy used in this study, AX52 is different in chemical composition from AZ91, the reported value is in good agreement with our result. Therefore, we conclude that the modified WarrenAverbach analysis is effective because of its simplicity and no arbitrary input data compared to the MWP fitting method.

\section{Conclusion}

The modified Warren-Averbach analysis method was revised and applied to the estimation of the dislocation density value in AX52 (Mg-Al-Mn-Ca) die-cast alloy from $\mathrm{XRD}$ profiles, and the validity of this analysis method was 
discussed. We found that this method is credible and accurate. A similar procedure using AX52 (Mg-Al-Mn-Ca) die-cast alloy in this study is also applicable to other hexagonal crystals, such as $\mathrm{Ti}, \mathrm{Zr}$ and $\mathrm{Zn}$, as well as MWP fitting.

\section{Acknowledgement}

This work was supported by Grant-in-Aid for Scientific Research of Japan Society for the Promotion of Science (JSPS), Japan.

\section{REFERENCES}

1) D. Itoh, Y. Terada and T. Sato: Mater. Trans. 49 (2008) 1957-1962.

2) G. Ribárik, T. Ungár and J. Gubicza: J. Appl. Cryst. 34 (2001) 669676.

3) K. Máthis, K. Nyilas, A. Axt, I. Dragomir-Cernatescu, T. Ungár and P.
Lukáč: Acta Mater. 52 (2004) 2889-2894.

4) K. Máthis, J. Gubicza and N. H. Nam: J. Alloy. Compd. 394 (2005) 194-199.

5) I. C. Dragomir, D. S. Li, G. A. Castello-Branco, H. Garmestani, R. L. Snyder, G. Ribárik and T. Ungár: Mater. Charact. 55 (2005) 66-74.

6) T. Ungár, M. G. Glavicic, L. Balogh, K. Nyilas, A. A. Salem, G. Ribárik and S. L. Semiatin: Mater. Sci. Eng. A 493 (2008) 79-85.

7) T. Ungár, O. Castelnau, G. Ribárik, M. Drakopoulos, J. L. Béchade, T. Chauveau, A. Snigirev, I. Snigireva, C. Schroer and B. Bacroix: Acta Mater. 55 (2007) 1117-1127.

8) T. Ungár, J. Gubicza, G. Ribárik and A. Brobély: J. Appl. Cryst. 34 (2001) 298-310.

9) I. C. Dragomir and T. Ungár: J. Appl. Cryst. 35 (2002) 556-564.

10) T. Ungár and G. Tichy: Phys. Status. Solid. 171 (1999) 425-434.

11) T. Ungár and A. Borbély: Appl. Phys. Lett. 69 (1996) 3173-3175.

12) T. Kunieda, M. Nakai, Y. Murata, T. Koyama and M. Morinaga: ISIJ Int. 45 (2005) 1909-1914.

13) T. Ungár, J. Gubicza, G. Ribárik and A. Borbély: J. Appl. Cryst. 34 (2001) 298-310.

14) Y. Murata, I. Nakaya and M. Morinaga: Mater. Transact. 1 (2008) 20-23. 